Behavior and Social Issues, 21, 152-164 (2012). (c) Angela K. Fournier \& Thomas D. Berry. Readers of this article may copy it without the copyright owner's permission, if the author and publisher are acknowledged in the copy and the copy is used for educational, not-for-profit purposes. doi: 10.5210/bsi.v.21i0.3979

\title{
EFFECTS OF RESPONSE COST AND SOCIALLY-ASSISTED INTERVENTIONS ON HAND-HYGIENE BEHAVIOR OF UNIVERSITY STUDENTS
}

\author{
Angela K. Fournier ${ }^{1}$ \\ Bemidji State University \\ Thomas D. Berry \\ Christopher Newport University
}

\begin{abstract}
A field study was conducted to examine the effect of interventions to increase hand-hygiene behavior of university students. Student patrons of a university cafeteria were observed during lunch. Across several phases, researchers observed and recorded the number of students (a) entering the restroom to wash hands and (b) using a hand-sanitizer gel. Interventions included an informational poster, hand-sanitizer dispenser, and change agent to increase hand-hygiene behavior. Results showed that the presence of a strategically placed hand-sanitizer dispenser was effective in increasing hand-hygiene behavior from $1.52 \%$ to over $60 \%$ (average $n=208$ students per day). Participants were particularly responsive to the hand-sanitizer dispenser when combined with a change agent. Meanwhile, the tested interventions were ineffective in increasing the number of students entering a restroom to hand wash. The results are discussed regarding response cost and socially-mediated consequences of change agents.

KEYWORDS: hand hygiene, hand-sanitizer gels, hand washing, change agent interventions, social assistance

There is a preponderance of research showing hand-washing behavior is a significant means of reducing pathogen exposure, spread and infection (see Aiello, Coulborn, Perez, \& Larson, 2008; Bryan, Cohran, \& Larson, 1995). Health experts are quick to point out the distinction between hand washing associated with personal consequences, and the societal implication of our hands as pathogen carriers to others (vectors for the spread of disease). At a societallevel, hand-borne pathogens cost the United States at least 23 million school-day

\footnotetext{
${ }^{1}$ Correspondence concerning this manuscript should be addressed to Angela Fournier, who is at the Department of Psychology, Bemidji State University, Bemidji, MN 56601. E-mail may be sent to afournier@bemidjistate.edu. Telephone inquiries may be made to 218-755-2530.
} 
absences, 25 million work-day absences, 27 million doctor visits, and $\$ 2$ billion in medications annually (Turner, 1998). For instance, our hands pick up, deposit, and share with others a whole host of hand-borne illnesses, such as Rhinovirus, Rotavirus, E-coli, Shigella, Hepatitis-A, and Salmonella, resulting in gastrointestinal and respiratory infections (GI and RI, respectively). It has been estimated that $60 \%$ of GI and $50 \%$ of RI suffered by society are the result of hand-shared and spread disease (Bloomfield, Aiello, Cookson, O'Boyle, \& Larson, 2007).

As stated by the CDC (1987), universal precautions (UP) to control nosocomial infection rates should incorporate an effective hand-washing repertoire. Furthermore, hand washing is defined as wetting the hands with clean running water, applying soap, rubbing the hands together to create lather, and rinsing the hands under clean, running water (CDC, 2011). Although this definition is an accepted means to minimize pathogen spread and infection, the definition offers little in behavior analytic considerations for the stimulus control and maintenance of hand washing. The research reported here targets hand hygiene from an environmental and behavioral intervention approach, seeking conditions relevant for the control and quality of hand hygiene.

Taking an environmental perspective, Venkatesh, Pallin, Kayden and Schuur (2011) studied a hospital's emergency department (ED) and found hand washing was more frequent when patients were located in rooms $(90.8 \%$ adherence) rather than in the hallway ( $82.3 \%$ adherence), and when the patient rooms were visible from the nursing station rather than obscured. Venkatesh et al. speculated that lower rates of hand washing for hallway patients were due to ED overcrowding and the accessibility of sinks. Consistent with Venkatesh et al., Kaplan and McGuckin's (1986) surveillance of medical and surgical ICUs where sink availability was differentiated found higher frequencies of hand washing when bed to sink ratios were 1:1 (76\% adherence) as opposed to $4: 1$ (51\% adherence). In fact, across the hand washing literature sink availability appears to be a key factor for cueing hand washing. These results are all the more important in the light of validity studies. For example, Chaudhury, Mahmood and Valente (2005) found that when a 1:1 bed-sink ratio existed, the frequency of nosocomial infections were significantly lowered.

Investigators have tested a number of behavioral interventions that influence hand-hygiene behavior, including signs and prompts (Johnson, Sholcosky, Gabello, Ragni, \& Ogonosky, 2003), education and awareness sessions (Columbine \& Wharrad, 2007), and feedback and goal setting (Babcock, SulzerAzaroff, Sanderson, \& Scibek, 1992: Luke \& Alavosius, 2011; Stephens \& Ludwig, 2005). Much of this research suggests that increases in hand-hygiene 
practices are relatively sensitive to immediate personalized feedback and to some extent the introduction of signs and prompts. It is important to note that intervention studies, as discussed, present participants a situation where sinks are presumed accessible, and/or incorporate a broad definition of hand hygiene that includes both hand washing and the use of hand-sanitizer gels. This broad hand hygiene definition is consistent with CDC recommendations (Boyce \& Pittet, 2002).

Hand-sanitizer gels or rubs have been well researched for their antipathogenic results, ease of use, and accessibility (e.g., Dyer, Gerenratch, \& Wadhams, 1998).). In fact, investigators have shown through modeling strategies that nursing staff using hand sanitizer exclusively would save time without sacrificing the quality of patient care (Voss \& Widmer, 1997). This research suggests that $100 \%$ hand-washing compliance by nurses would take $17 \%$ of their total working time (Voss \& Widmer, 1997; Widmer, 2000). In contrast, nurses that switched to using hand-sanitizer gels would take $<3 \%$ of their total working time. The inclusion of hand-sanitizer gels, as part of the hand-hygiene definition, underscores the behavioral importance of hand sanitizer's ease and accessibility of use sufficient for improving and maintaining hand-hygiene practices.

Since the outbreak of SARS and H1N1, college student hand-hygiene practices have grown in importance and concern. Consistent with the above, White et al. (2003) showed that students provided with easy and convenient access to hand-sanitizer gels were significantly less likely than a control group of students to suffer symptoms, illness, and absenteeism. Over a six-week period of study, Aiello et al. (2012) found that hand sanitizers coupled with facemask use reduced influenza-like illness between $48 \%$ and $75 \%$ per week as compared to a control group of residential-based students. The research presented below targeted university students' hand sanitizer use as associated with a dining hall setting during the lunch hour.

\section{Pilot Study}

Our research began with a pilot study attempting to approximate natural frequency of hand washing of students prior to eating their lunch at a college cafeteria. Researchers made 4,144 observations over 20 days, observing individual students from the time they entered the cafeteria until they began eating their food. These pilot observations revealed that the baseline frequency of students entering a restroom to hand wash demonstrated a floor effect. During the pilot observations interventions were implemented, including an (a) informational 
poster, and (b) informational leaflet ${ }^{2}$ handed out by a female student serving as a change agent. Both the poster and leaflet provided information about pathogen risk and encouraged hand washing. However, during the intervention phases, the number of students observed entering the restroom to hand wash did not change. Interestingly, the behavior change techniques used in our pilot research have been previously shown to influence student hand hygiene (e.g., Surgeoner, Chapman, \& Powell, 2009), the hand hygiene of food preparation staff (e.g., Geller, Eason, Phillips, \& Pierson, 1980) as well as other important health behaviors (e.g., Berry, Geller, Calef, \& Calef, 1992; Williams, Thyer, Bailey, \& Harrison, 1989). However, university students in this cafeteria setting were not influenced by these traditional intervention techniques. A potential reason for this non-effect points to the response-cost associated with students leaving their place in line in order to enter the restroom, access a sink, and wash their hands.

Thus, we examined an alternative to traditional hand washing; specifically we studied the use of hand-sanitizer gel. Hand-sanitizer gels that contain $60 \%$ to $70 \%$ ethyl alcohol have both bacterial and viral killing activity (CDC, 2010; Willis, 1995). Therefore, ethyl alcohol is an ideal hand cleaning method, particularly for busy university students, because in addition to disinfecting hands, it can be easily carried in a person's pocket, brief case or purse; does not require a restroom or faucet and sink; and applying hand sanitizer can take place almost anywhere and anytime. The main goal was to assess whether students would access handsanitizer gel, and which intervention conditions influence the use of hand sanitizer.

\section{Method}

\section{Participants and Setting}

Participants included college men and women eating at a residential cafeteria on a university campus in mid-Atlantic United States. The cafeteria served one dorm of 450 college students and was attached to the dorm by a covered breezeway. Figure 1 shows the cafeteria had a single entrance/exit location, one cashier counter, one serving line for food, and an open eating area with tables and chairs. The cafeteria was one large room with an open floor plan, so that one could view the entire space from the back of the room. This open floor plan made it possible to observe patrons entering, paying, entering/exiting the restrooms, walking through the serving line, and finally sitting down to eat. The cafeteria had

\footnotetext{
${ }^{2}$ A copy of the leaflet can be acquired by contacting the first author.
} 
one men's restroom and one women's restroom. The men's restroom had one urinal, one stall, and one sink. The women's restroom had two stalls and one sink. On entering the dining hall, students were required to pay a cashier before going to the cafeteria-style serving lines. Students would pass the two restroom doorways after leaving the cashier and before entering the serving lines. These restrooms were the only locations where students could wash their hands prior to eating. During the intervention phases, a round table outfitted with a dispenser of

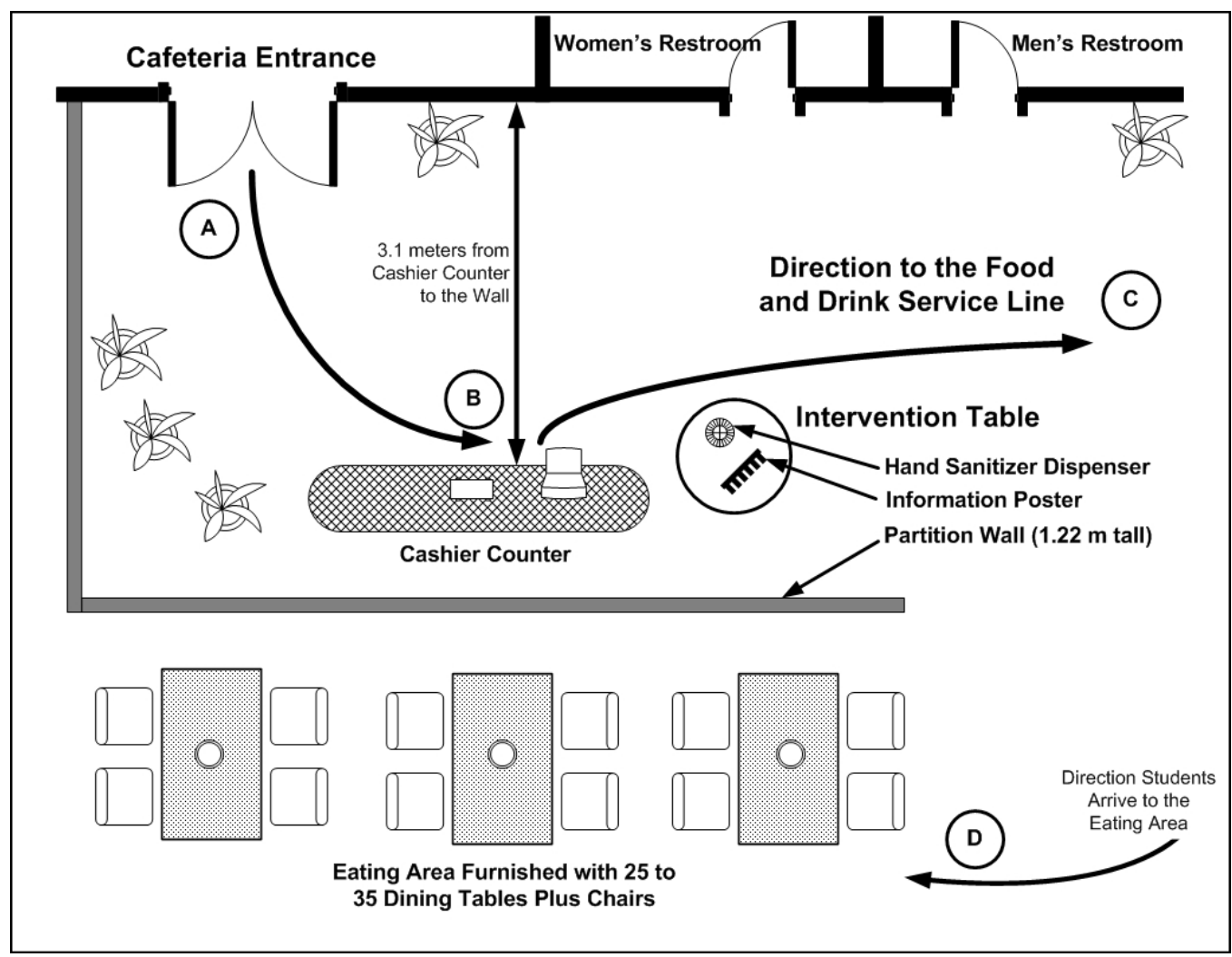

Figure 1. This schematic shows a portion of the student-cafeteria layout and the typical path students followed: (A) from the Entrance, (B) to the Cashier Counter, (C) pass by Women's and Men's Restrooms toward the Food and Drink Service Line, and (D) after selecting food and drink students proceed to the Eating Area. Also shown is the general location of the round Intervention Table where the Change Agent stood and prompted hand sanitizer use. Additionally, the layout indicates the distance measured from the Cashier Counter to the opposite wall (i.e., $3.1 \mathrm{~m}$ ) and where the Restrooms were co-located. 
hand-sanitizer gel and an information poster was placed next to the cashier counter. After leaving the serving line with their food and drink students moved to the open commons area where tables and chairs were located.

\section{Procedure}

Observations took place during spring semester in the cafeteria at lunch time from $12 \mathrm{pm}$ to $1 \mathrm{pm}$. Data were collected Monday through Friday for four consecutive weeks. Student observers sat at a side table in the eating area with their lunches. Observers unobtrusively collected the following data: (a) number and gender of students entering the dining hall, and (b) number and gender of students entering the dining hall restrooms.

When the hand-sanitizer dispenser was first implemented, two observers recorded the number of students who operated the hand-sanitizer dispenser. One observer sat unobtrusively in the dining hall at a table with her lunch. The other observer was a change agent who stood by the table and dispenser. She was instructed to stand next to the table and dispenser and ask students if they wanted to clean their hands, kill germs, and avoid getting sick by using the hand sanitizer. The change agent operated a hand held four-digit counter, clicking it once for each student using the dispenser. The change agent was instructed to make counts discreetly and to keep the hand-held counter hidden from students. She did not collect any other type of data.

\section{Study Design and Interventions}

The study followed a five phase A-BC-B-BC-B design with phases, lasting three to five days, defined as:

Baseline (A). During the first phase, lasting three days, the number of patrons entering the restrooms to hand wash was observed without intervention. To compare the future intervention effectiveness of using hand sanitizer, students' use of personal hand sanitizers was observed. Observations began when students entered the cafeteria line and ended when they began eating their lunch.

Hand sanitizer, information poster, and change agent (BC). During phases two and four, a round table was placed between the cashier and serving line (see Figure 1). On the table was placed a white one-liter ceramic dispenser filled with the hand sanitizer, a $62 \%$ ethyl alcohol gel. To dispense the hand sanitizer, a user was required to press down on a spout-activated pump mechanism once, collect the hand sanitizer in the hand, and rub the hands together back and forth for several seconds until the hand sanitizer had covered the hands and dissolved. Each press of the pump released a quarter size amount of hand 
sanitizer, approximately 2.50 grams. Accompanying the table and dispenser was (a) a white $61.4 \mathrm{~cm} \times 92.2 \mathrm{~cm}$ poster with the wording, "Sanitize your hands to prevent cold and flu," (b) a sign attached to the ceramic dispenser labeling it "Hand Sanitizer," and (c) a change agent that encouraged and educated students about hand-sanitizer gels.

Hand sanitizer and information poster (B). Phases three and five were the same as above except that the change agent was removed from the intervention setting.

\section{Results}

\section{Interobserver Reliability}

Interobserver reliability was calculated for students' use of the hand-sanitizer dispenser. During BC and B phases, hand sanitizer use was observed 9 out of 17 days by two independent observers (i.e., the change agent and an observer in the dining area). For each day of the study, percent agreement was calculated by dividing the number of observations in which the observers were in agreement by the total number of observations. Mean percent agreement for number of students using the hand sanitizer was $94.5 \%$, ranging from $88 \%$ to $99 \%$.

\section{Overall Effects}

A total of 6,454 observations were recorded across seven consecutive weeks, including 3,226 men and 3,228 women. The average number of observations per day was 208, ranging from 151 to 282 . There were no significant gender differences in hand-hygiene behavior, $p>.10$. Therefore, all data represent observations from men and women combined. Additionally, during the baseline phase (A phase) students' use of their own personal hand sanitizers was observed. No students were observed using their own personal hand sanitizer.

Differences between phases are illustrated in Figure 2, showing a substantial increase in hand-hygiene behavior from baseline to the implementation of the interventions. During baseline (A Phase) observations indicated that students did not use personal hand sanitizer $(M=0 \%)$, while the introduction of the handsanitizer dispenser, information poster, and change agent (BC Phase) showed a dramatic increase $(M=60.44 \%)$ of student use of hand sanitizer from the dispenser provided. Removal of the change agent from the table outfitted with the information poster and the hand-sanitizer dispenser (B Phase) showed a substantial decrease in student use of the hand sanitizer $(M=17.96 \%)$. In phase four, subsequent return of the change agent (BC Phase) saw another robust 


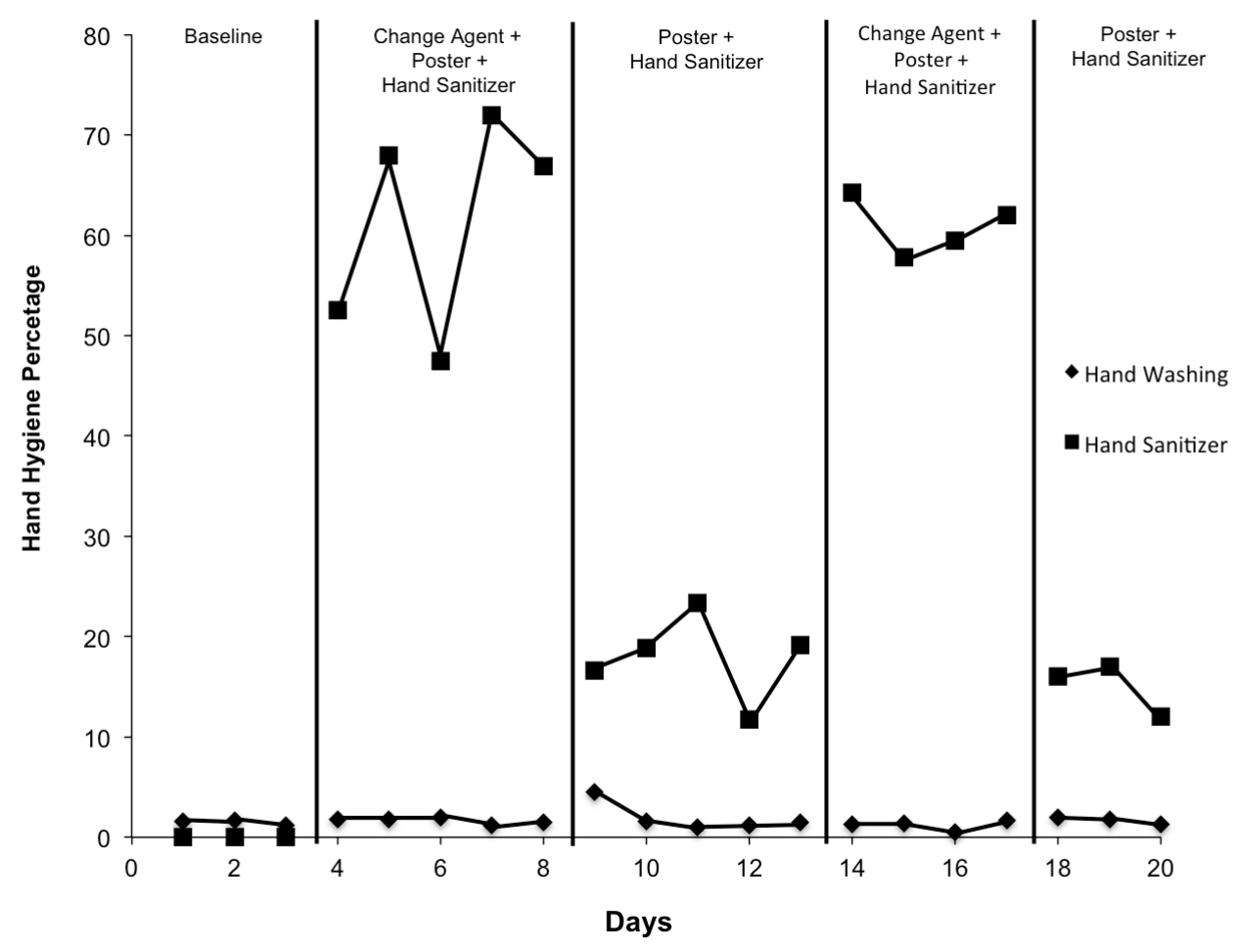

Figure 2. Percentage of students engaging in hand washing and hand sanitizing behavior across each phase of the study.

increase in hand sanitizer use $(M=60.96 \%)$. Finally, in phase five the removal of the change agent (B Phase) saw a large decrease in student use of the hand sanitizer $(M=14.83 \%)$. Additionally, as can be seen in Figure 2, the number of students entering the women's or men's restroom was unchanged across all phases $(M=1.56 \%)$. Thus, the interventions presented presumably did not influence students to wash their hands with soap and water in these restrooms.

\section{Discussion}

The present study suggests that a strategically-placed dispenser of hand sanitizer may prove to be an effective method of increasing student hand hygiene. Although the use of hand sanitizer as compared to hand washing represents different behaviors, both operate to clean hands by removing or killing pathogens. 
The interventions presented here resulted in robust increases in hand hygiene, with both the hand sanitizer presented alone $(M=17.96 \%)$ and with a change agent $(M=60.96 \%)$. In both cases, the hand-sanitizer dispenser was placed in the dining hall where it did not interrupt the natural flow of students' accessing lunch lines. In one sense, the hand-sanitizer dispenser became an additional but inserted part of a chain of behaviors leading to food service (see Figure 1). Because of dispenser placement, students (a) did not have to leave the area to clean their hands in a restroom, (b) did not lose their place in the serving line, and (c) dispensing of hand sanitizer was quick and easy, a task taking a few seconds. In addition, the present findings are consistent with large-scale behavioral research showing that a prompt occurring in closer proximity to the opportunity to emit the behavior, also known as "point-of-purchase advertising," can be more effective than a more distal prompt (Geller, Farris, \& Post, 1973).

Baseline hand washing may have been avoided because of the inconvenience or response cost of taking the time and effort. Students often go to lunch with friends, and thus entering the restroom to wash one's hands means separating from lunch mates and breaking from the serving line. Similar barriers have shown to decrease intentions to hand wash by medical staff, including such significant predictors as time inconvenience, number of sinks, sink location and availability (Jenner, Watson, Miller, Jones, \& Scott, 2002). This factor of time and inconvenience may explain why students who did not enter the restroom to wash their hands did access the hand-sanitizer dispenser.

The presence or absence of a change agent was reflected in different levels of hand sanitizing. In general, there was a more than three-fold increase in hand sanitizing when a change agent was standing next to the hand-sanitizer dispenser and information poster as compared to the dispenser and poster alone condition. However, the relative contribution made by each component to observed increases in hand hygiene must be considered with caution. The potential serial dependency may have obscured our understanding of how hand sanitizer presented without a change agent might influence student use. The justification of the current design was based on practical reasons. Given the results of our pilot study that revealed low rates of students entering the restrooms to wash their hands, and (more importantly) student insensitivity to interventions, a treatment package of multiple components was deemed appropriate (see Azrin, 1977; Williams et al., 1989 for an example).

Because data collection was strictly observational, researchers were unable to question participants about the effects of the interventions, particularly the differential impact of the hand sanitizer with and without the change agent. Beyond an ordering effect, the increased hand-hygiene behavior with a change 
agent may be understood in the context of social assistance. Social assistants function presumably by providing socially-mediated consequences (e.g., approval, respect, and avoidance of negative admonishment). Socially-assisted interventions have been found to improve safety-related behavior over and above the impact of the intervention alone (e.g., Berry et al., 1992; Williams et al., 1989). Social assistance is particularly useful when the natural contingencies governing behavior are non-direct acting (Malott, 1988, 1989).

The consequence of pathogen infection due to hand-born disease is likely a non-direct acting contingency with weak stimulus control properties. Pathogen risk is not easily detectable by people. Where, when and what pathogens are present, day to day, in the world of objects (e.g., doors, elevator buttons, ATMs and so on) adds to the probabilistic nature of pathogen risk. Thus, it is difficult for a person to determine how and where he or she became exposed and infected by a disease (Ansari, Sattar, Springthorpe, Wells, \& Tostowaryk, 1988). Without reliable natural cues and direct acting consequences, establishing hand washing as a prophylactic response to pathogen risk will likely be difficult.

Malott (1988, 1989) suggests that non-direct acting contingencies be supplemented with indirect acting contingencies, defined as establishing sociallycontrived consequences. During the present research, the change agent's association with the hand-sanitizer dispenser showed remarkable influence over hand-hygiene behavior. As mentioned in the Method section, the change agent was instructed to educate students about hand-sanitizer gels, and encourage students to use the sanitizer dispenser. The interactions between the change agent and students over the course of the phases may have provided supplemental social consequences important to the hand sanitizer's effectiveness.

However, the results suggest effectiveness of the change agent may be dependent on other intervention characteristics. In our pilot research, as mentioned in the introduction, a change agent was employed to distribute leaflets that stated the importance of hand washing before eating lunch. During this condition, the change agent, leaflet and poster intervention had no effect on students entering the cafeteria restrooms to hand wash. The difference between the pilot study change agent effectiveness and the present study may be due to the change agent's proximity to the hand-hygiene behavior (i.e., point-of-purchase advertising, Geller et al., 1973). In the present study, both students and the change agent were in close proximity to the sanitizer dispenser, and use of the dispenser was in full view of the change agent. Thus, the change agent was able to apply immediate social consequences for hand hygiene. In contrast, in the pilot study the change agent's ability to give social consequences was impossible due to the distant location of the restroom and subsequent hand washing. 
In terms of research feasibility, the use of hand sanitizer was not financially prohibitive. Combining the four phases in which sanitizer was used, it cost less than $\$ 30.00$ to dispense the product to 1,375 students over 17 days. Likewise, the cost of the signs was minimal, less than $\$ 25.00$. The greatest expense would be associated with the change agent. Although the change agent was an unpaid research assistant, her average hourly routine included 2 hours of work per day. Alternatively, it may be possible for a trained cafeteria cashier to also serve as a change agent, offering students hand sanitizer after paying for their food.

Several limitations of this research are noteworthy. First, measurements of positive health benefits from increasing hand hygiene were not collected. At the time of this research no records were available to correlate health benefits to student hand-hygiene behavior. Second, students studied were of a restricted age group. Intervention effectiveness may show different results across a more diverse and mature group of people. Third, individual student behavior was not tracked and measured over time. Thus, we were unable to observe the strength of the intervention(s) to control individual variation (e.g., Berry, Gilmore, \& Geller, 1994). Fourth, although change agent plus hand sanitizer and hand sanitizer alone showed increases in hand hygiene, the shortness of the phases (i.e., 3 to 5 days) did not allow for observing long-term maintenance and trends. Future research is encouraged to target issues of hand hygiene maintenance and trends, along with associated health measures to examine long-term health benefits. Research on university student hand hygiene is clearly needed to better ascertain the effectiveness and viability of interventions to influence student hand washing and hand hygiene practices.

\section{References}

Aiello, A. E., Coulborn, R. M, Perez, V., \& Larson, E. L. (2008). Effect of hand hygiene on infectious disease risk in the community setting: A meta-analysis. American Journal of Public Health, 98(8), 1372-1381. doi: 10.2105/AJPH.2007.124610

Aiello, A. E., Perez, V., Coulborn, R. M, Davis, B. M., Uddin, M., \& Monto, A. S. (2012). Facemasks, hand hygiene, and influenza among young adults: A randomized intervention trial. PLOS One, 7(1), e29744. doi: 10.1371/journal.pone.0029744

Ansari, S. A., Sattar, S. A., Springthorpe, V. S., Wells, G. A., \& Tostowaryk, W. (1988). Rotavirus survival on human hands and transfer of infectious virus to animate and nonporous inanimate surfaces. Journal of Clinical Microbiology, 26, 1513-1518.

Azrin, N. H. (1977). A strategy for applied research: Learning based but outcome oriented. American Psychologist, 33, 140-149.

Babcock, R., Sulzer-Azaroff, B., Sanderson, M., \& Scibek, J. (1992) Increasing nurses' use of feedback to promote infection control practices in a head injury treatment center. Journal of Applied Behavior Analysis, 25, 621-627. 


\section{HAND-HYGIENE BEHAVIOR}

Berry, T. D., Geller, E. S., Calef, R. S., \& Calef, R. S. (1992). Moderating effects of social assistance on verbal interventions to promote safety belt use: An analysis of weak plys. Environment and Behavior, 24, 653-669.

Berry, T. D., Gilmore, M., \& Geller, T. D. (1994). An individual-subject approach to the study of community-based interventions. Environment and Behavior, 26, 451-476.

Bloomfield, S. F., Aiello, A. E., Cookson, B., O'Boyle, C., \& Larson E. L. (2007). The effectiveness of hand hygiene procedures, including handwashing and alcohol-based hand sanitizers, in reducing the risks of infections in home and community settings. American Journal of Infection Control, 35 (10), 27-64.

Boyce, J. M., \& Pittet, D. (2002, October 25). Guideline for hand hygiene in health-care settings: Recommendations of the healthcare infection control practices advisory committee and the HICPAC/SHEA/APIC/IDSA hand hygiene task force. Retrieved from http://www.cdc.gov/MMWR/preview/mmwrhtml/rr5116a1.htm

Bryan, J. L., Cohran, J., \& Larson, E. L. (1995). Hand washing: A ritual revisited. Infection and Control in Critical Care, 7 (4), 617-625.

Centers for Disease Control and Prevention. (1987). Universal precautions for prevention of transmission of HIV and other bloodborne infections. Fact Sheet. Retrieved from http://www.cdc.gov/ncidod/dhqp/bp_universal_precautions.html

Centers for Disease Control and Prevention. (2010, August 24). Norovirus: Technical fact sheet. Retrieved from http://www.cdc.gov/ncidod/dvrd/revb/gastro/norovirus-factsheet.htm

Centers for Disease Control and Prevention. (2011). Handwashing: Clean hands save lives. Retrieved from http://www.cdc.gov/handwashing/

Chaudhury, H., Mahmood, A. \& Valente, M. (2005). Advantages and disadvantages of single vs. double occupancy patient rooms in acute care environments: A review and analysis of the literature. Environment and Behavior, 37(6), 760-786.

Columbine, A., \& Wharrad, H. (2007). Using computer technology to deliver an infection control update on hand hygiene. British Journal of Infection Control, 8 (3), 14 - 19.

Dyer, D. L., Gerenratch, K. B., \& Wadhams, P. S. (1998). Testing a new alcohol-free hand sanitizer to combat infection. AORN Journal, 68 (2), 239-251.

Geller, E. S., Eason, S., Phillips, J., \& Pierson, M. (1980). Interventions to improve sanitation during food preparation. Journal of Organizational Behavior Management, 2, 229-240.

Geller, E. S., Farris, J. C., \& Post, D. S. (1973). Prompting consumer behaviors for pollution control. Journal of Applied Behavior Analysis, 6, 345-353.

Jenner, E. A., Watson, P. W. B., Miller, L., Jones, F., \& Scott, G. M. (2002). Explaining hand hygiene practice: An extended application of the theory of planned behavior. Psychology, Health \& Medicine, 7 (3), 311-326. doi: 10.1080/13548500220139412

Johnson, H. D., Sholcosky, D., Gabello, K., Ragni, R., \& Ogonosky, N. (2003). Sex differences in public restroom handwashing behavior associated with visual behavior prompts. Perceptual and Motor Skills, 97 (3), 805-810.

Kaplan, L. M., \& McGuckin, M. (1986). Increasing handwashing compliance with more accessible sinks. Infection Control, 7 (8), 408-410.

Stephens, S. D., \& Ludwig, T. D. (2005). Improving anesthesia nurse compliance with universal precautions using group goals and public feedback. Journal of Organizational Behavior Management, 25(2), 37-71.

Luke, M., \& Alavosius, M. P. (2011). Adherence with universal precautions after immediate, personalized performance feedback. Journal of Applied Behavior Analysis, 44 (4), 967-971. 


\section{FOURNIER \& BERRY}

Malott, R. W. (1988). Rule-governed behavior and behavioral anthropology. The Behavior Analyst, 11(2), 181-204.

Malott, R. W. (1989). The achievement of evasive goals: Control by rules describing contingencies that are not direct acting. In S. C. Hayes (Eds.), Rule-governed behavior: Cognitions, contingencies, and instructional control (pp. 269-322). New York: Plenum Press.

Surgeoner, B. V., Chapman, B. J., \& Powell, D. A. (2009). University students' hand hygiene practice during a gastrointestinal outbreak in residence: What they say they DO and what they actually do. Journal of Environmental Health, 72(2), 24-28. Retrieved from http://www.ncbi.nlm.nih.gov/pubmed/19761005

Turner, R. B. (1998). The common cold. Pediatric Annals, 27 (12), 790-795.

Venkatesh, A. K., Pallin, D. J., Kayden, S., \& Schuur, J. D. (2011). Predictors of hand hygiene in the emergency department. Infection Control and Hospital Epidemiology, 32 (11), 11201123.

Voss, A., \& Widmer, A. F. (1997). No time for handwashing, can we achieve $100 \%$ compliance? Infection Control of Hospital Epidemiology, 18 (3), 205-208.

Widmer A. F. (2000). Replace hand washing with use of a waterless alcohol hand rub? Clinical Infectious Diseases, 31 (1), 36-43.

Williams, M., Thyer, B. A., Bailey, J. S., \& Harrison, D. F. (1989). Promoting safety-belt use with the traffic signs and prompters. Journal of Applied Behavior Analysis, 22, 71-76.

Willis, J. (1995). Skin care: Principles of hand-washing. Nursing Times, 91 (44), 43-44.

White, C., Kolbe, R., Carlson, R., Lipson, N., Dolan, M., Yusuf, A., \& Cline, M. (2003). The effect of hand hygiene on illness rate among students in university residence halls. American Journal of Infectious Control, 31(6), 364-370. doi: 10.1177/0013916511408069 